\title{
Novel Regulated Secretion Mechanism For a Nerve-Secreted Cell Signaling Molecule in C. elegans
}

\author{
R.D. Schultz ${ }^{1,2}$, K. Beifuss ${ }^{1}$, S. Bageshwar ${ }^{1}$, E.A. Ellis ${ }^{3}$, and T.L. Gumienny ${ }^{1,2,4}$ \\ ${ }^{1}$ Molecular and Cellular Medicine, Texas A\&M University Health Science Center, College Station, TX, \\ USA. \\ ${ }^{2}$ Interdisciplinary Program in Genetics, Texas A\&M University, College Station, TX, USA \\ ${ }^{3}$ Microscopy and Imaging Center, Texas A\&M University, College Station, TX, USA. \\ ${ }^{4}$ Department of Biology, Texas Woman's University, Denton, TX, USA.
}

Bone morphogenetic proteins (BMPs) are cell-cell signaling molecules that are conserved from sea urchins to vertebrates [1]. The BMP family of ligands is dose-dependent and must undergo complex means of secretion, spatial regulation, and degradation to regulate processes such as extracellular growth and homeostasis. The receptors and signaling pathways that this protein family stimulates is well characterized, but the mechanisms controlling how much of the signal is released are unknown [2]. The free-living nematode $C$. elegans is an ideal system in which to study this cellular BMP regulatory process, with its conserved molecular pathways, ease of genetic manipulation, and simple, transparent body for visualizing fluorescently tagged transgenic proteins.

The C. elegans BMP member DBL-1 is expressed in nerves and activates receptors on surrounding epidermal tissue [3]. Some nerve-secreted proteins can be secreted constitutively, without the help of vesicle transport, while other nerve-secreted proteins undergo regulated secretion, using synaptic or dense core secretory vesicles to be transported from the Golgi to the cell's plasma membrane [4]. Using a green fluorescent protein (GFP)-tagged DBL-1, we show that DBL-1 clusters in vesicle-sized punctae in neurons (Figure 1). This suggests that secretion of DBL-1 is regulated. A functional mammalian BMP4 expressed in C. elegans and visualized using a novel, microwave-based immunocytochemical procedure is also punctate and co-localizes with $C$. elegans DBL-1 (Figure 1). To determine the nature of these punctae, we examined for co-localization between either BMP4 or GFP-tagged DBL-1 and various vesicle components. While we did not find any evidence that DBL-1 is dependent on synaptic or dense core vesicle secretion, we discovered that BMP4 co-localizes at the neuronal plasma membrane with dynamin and caveolin (Figure 2). While caveolin is best known for its role in endocytosis and signal transduction, limited findings suggest caveolin transport from the Golgi to the plasma membrane can include cargo [5]. We found that DBL-1 not only co-localizes with caveolin, but loss of caveolin reduces DBL-1 levels and signaling. Using time-lapse microscopy, we found a sub-population of DBL1-positive punctae move in a manner consistent with microtubule-based transport (Figure 3). This directed transport of DBL-1 further supports a role for caveolin in DBL-1 secretion, as some caveolin vesicles can move on microtubules.

Caveolin-associated vesicles have been shown to undergo "kiss-and-run" dynamics, where vesicles transiently fuse with the plasma membrane using dynamin, allowing a partial release of vesicle contents into the extracellular milieu [6]. We provide evidence that regulation of DBL-1 vesicle secretion may be provided through caveolin-based "kiss-and-run" dynamics, which could contribute to dose and spatial control of DBL-1 pathway signaling. We propose that C. elegans DBL-1/BMP signaling is regulated by a novel caveolin-dependent secretion mechanism that controls BMP release from neurons. 


\section{References}

[1] IL Blitz and KW Cho, Dev Dyn 238 (2009), p. 1321.

[2] MC Ramel and CS Hill, FEBS letters 586 (2012), p. 1929.

[3] TL Gumienny and C Savage-Dunn in "WormBook", ed. The C. elegans Research Community, doi/10.1895/wormbook.1.22.2.

[4] S Houy et al, Front Endocrinol 4 (2013), p. 135.

[5] RG Parton and K Simons, Nat Rev Mol Cell Biol 8 (2007), p. 185.

[6] L Pelkmans and M Zerial, Nature 436 (2005), p. 128.

[7] This work was supported by NIH 1R01GM097591-01 and by TAMHSC MCMD start-up funds.
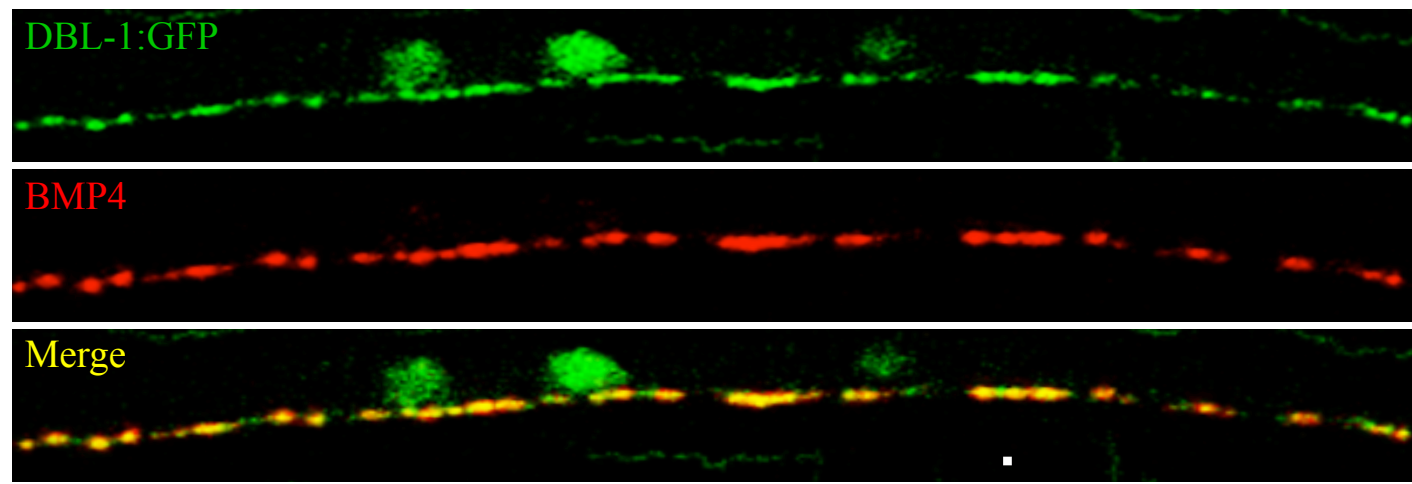

Figure 1. Fluorescent imaging of GFP-tagged DBL-1 (green) with immunolabelled BMP4 (red) shows protein co-localization in motor neurons. Scale bar $=5 \mu \mathrm{m}$.
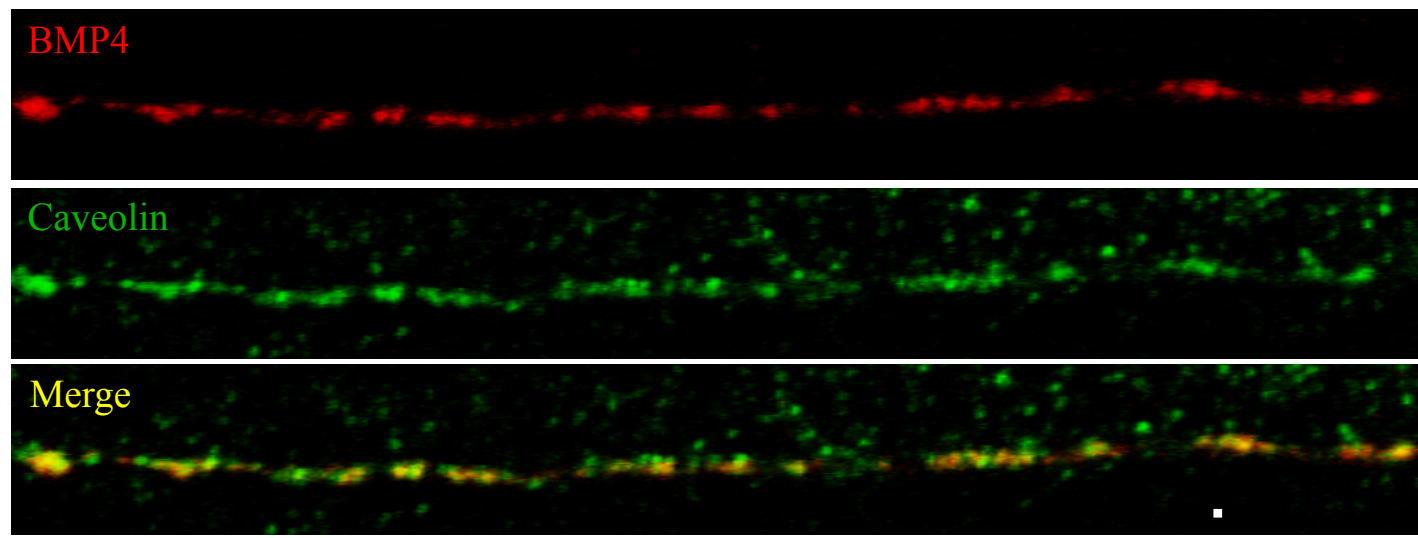

Figure 2. Fluorescent imaging of immunolabelled BMP4 (red) with caveolin (green) shows protein co-localization in motor neurons. Scale bar $=5 \mu \mathrm{m}$.
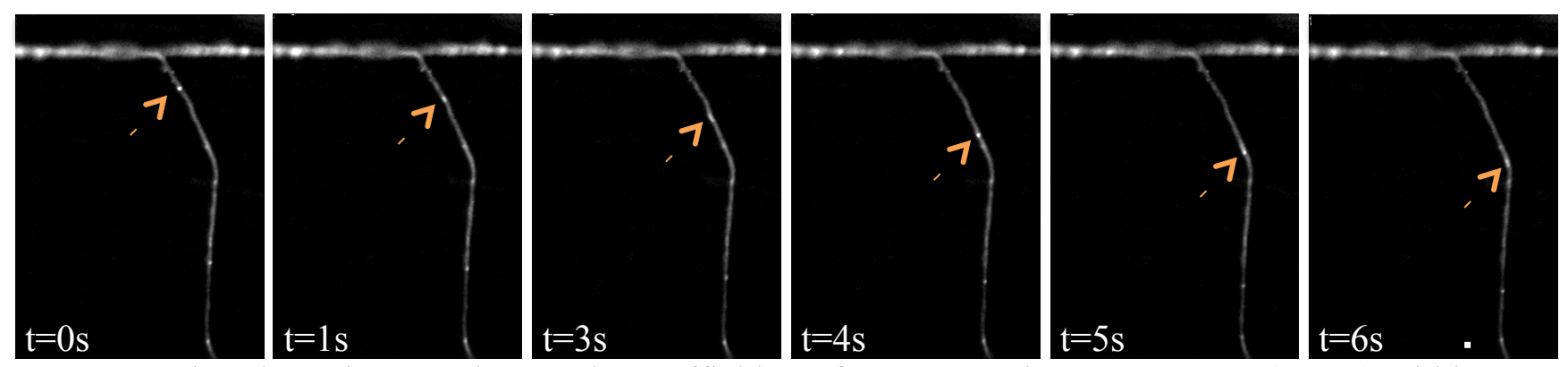

Figure 3. Time-lapse images show active trafficking of GFP-tagged DBL-1 puncta (arrow) within a nerve cell. Scale bar $=100 \mu \mathrm{m}$. 